\title{
Composite generalized elliptic curve-based surface reconstruction
}

Ouwen $\mathrm{Li}^{1}$, Ehtzaz Chaudhry ${ }^{1}$, Xiaosong Yang ${ }^{1}$, Haibin $\mathrm{Fu}^{1}$, Junheng Fang ${ }^{1}$, Zaiping Zhu', Andres Iglesias ${ }^{2}$, Algirdas Noreika ${ }^{3}$, Alfonso Carriazo ${ }^{4}$, Lihua You ${ }^{1}$, and Jian Jun Zhang ${ }^{1}$

${ }^{1}$ The National Center for Computer Animation, Bournemouth University, UK

2 Department of Applied Mathematics and Computational Sciences, University of Cantabria, Cantabria 39005, Spain

${ }^{3}$ Indeform Ltd, Lithuania

${ }^{4}$ Department of Geometry and Topology, University of Seville, Spain

\begin{abstract}
Cross-section curves play an important role in many fields. Analytically representing cross-section curves can greatly reduce design variables and related storage costs and facilitate other applications. In this paper, we propose composite generalized elliptic curves to approximate open and closed cross-section curves, present their mathematical expressions, and derive the mathematical equations of surface reconstruction from composite generalized elliptic curves. The examples given in this paper demonstrate the effectiveness and high accuracy of the proposed method. Due to the analytical nature of composite generalized elliptic curves and the surfaces reconstructed from them, the proposed method can reduce design variables and storage requirements and facilitate other applications such as level of detail.
\end{abstract}

Keywords: Curve modelling, composite generalized elliptic curves, surface modelling, composite generalized elliptic curve-based surface reconstruction, analytical mathematical expressions.

\section{Introduction}

Cross-section curves define two-dimensional contours of 3D objects. They have a lot of applications in many fields. Especially, they are used in medical imaging to reconstruct 3D models from cross sections for visualization.

Cross-sections obtained from imaging techniques or 3D polygon models are defined by discrete points, which involve many design variables, require high storage costs, cause slow network transmission, and do not meet the requirements of many applications such as level of detail where different resolutions are used in different situations.

In order to tackle the above problems, this paper will propose composite generalized elliptic curves, present their analytical mathematical expressions, and investigate surface reconstruction from composite generalized elliptic curves. Examples will be given to demonstrate the effectiveness and accuracy of the proposed method. 


\section{Related Work}

Various methods have been developed to reconstruct 3D models from cross sections called cross-section curves in this paper. Here, we briefly review some of them.

Early work on surface reconstruction from cross sections given in [1] found minimum cost cycles in a directed toroidal graph and presented a fast triangular tiling algorithm to reconstruct 3D surfaces consisting of triangular tiles from a set of cross-section curves. Using the contour information obtained from computed tomography, ultrasound examinations, and nuclear medicine examinations, the comparison among several triangular tiling algorithms are made in [2]. Through pruning the Delaunay triangulations obtained between two adjacent cross sections, a 3D polyhedron model was constructed whose triangular faces intersects with the planes along the given cross-sections [3]. Assuming that cross sections are perpendicular to the z-axis and reconstructed surfaces can be represented in cylindrical coordinates, fitting tensor product splines to the given cross sections with the least squares method was used to reconstruct open and closed 3D surfaces in [4]. A method was proposed in [5] to obtain a triangulated mesh from cross sections and a piecewise parametric surface was fitted to the triangulated mesh to produce a reconstructed surface. $3 \mathrm{D}$ reconstruction was achieved by decomposing one branching problem into several single-branching problems and using the closest local polar angle method to connect single branch contours [6]. A reconstructed surface was obtained in [7] by imposing three constraints on it, deriving precise correspondence and tiling rules from the constraints, and making the reconstructed surface produce the original contours. In order to avoid the problems caused by correspondence, tiling, and branching, surface reconstruction was formulated in terms of a partial differential equation and its solution was derived from the distance transform for dense contours and by adding a regulation term to ensure smoothness in surface recovery for sparse contours $[8,9]$. The Bernstein basis function (BBF) network, which is a two-layer basis function network, was used to fit closed parametric curves or contours to the edge points of planar images through the network training and reconstruct 3D surfaces from fitted parametric curves [10]. Using cross-section ellipses plus a displacement map for modelling and deformations of human arms and legs was given in [11]. The work in [11] was further extended to human deformations in [12]. Based on a stratification of polygons and anisotropic distance functions, an implicit surface was used to reconstruct a 3D model from cross sections, which avoid correspondence and branching problems [13]. A $G^{1}$ continuous composite surface consisting of skinned, branched, and capped surfaces was used in [14] to reconstruct 3D models where each of skinned surfaces was represented by a B-spline surface approximating cross sections and transformed into a mesh of rectangular Bezier patches and triangular $G^{1}$ surfaces were constructed in branched and capped regions. A new algorithm was proposed in [15] to deal with curve networks of arbitrary shape and topology on non-parallel cross-section planes with arbitrary orientations. How to provide topological control during surface reconstruction from an input set of planar cross-sections was investigated in [16]. A template model was used to better capture geometric features and deformed to fit a set of cross section curves in [17]. A globally consistent signed distance function was used in [18] to construct closed and smooth 3D surfaces from unorganized planar cross sections. Based on 
the idea of incremental sampling and potential field iterative correction, radial basis function and signed distance field based surface reconstruction is used in orebody modelling [19].

Different from the above work, in this paper, we will first propose analytical mathematical representations of cross-section curves called composite generalized elliptic curves including generalized ellipses, generalized elliptic curves, and composite generalized elliptic segments. Then we give analytical mathematical formulae for surface reconstruction from composite generalized elliptic curves.

\section{Generalized ellipses (GEs) and generalized elliptic curves (GECs)}

Elliptic cross sections have been used in sweeping surfaces to describe human arms, legs, torso and neck and their shape changes in [12] by Hyun et al. The elliptic cross section-based sweeping surfaces are mathematically formulated as [12]

$$
\begin{gathered}
\boldsymbol{S}(u, v)=\boldsymbol{R}(u) \boldsymbol{E}_{u}(v)+\boldsymbol{C}(u) \\
=\left[\begin{array}{lll}
r_{11}(u) & r_{12}(u) & r_{13}(u) \\
r_{21}(u) & r_{22}(u) & r_{23}(u) \\
r_{31}(u) & r_{32}(u) & r_{33}(u)
\end{array}\right]\left[\begin{array}{c}
a(t) \cos (v) \\
b(t) \sin (v) \\
0
\end{array}\right]+\left[\begin{array}{c}
x(u) \\
y(u) \\
z(u)
\end{array}\right]
\end{gathered}
$$

where $\boldsymbol{S}(u, v)$ represents a sweep surface, $\boldsymbol{R}(u)$ and $\boldsymbol{C}(u)$ are rotation and translation, respectively, and $\boldsymbol{E}_{u}(v)$ defines a standard ellipse of variable size.

Human bodies have irregular cross-section shapes. They cannot be well approximated with standard ellipses, which lead to unrealistic human body modelling as shown in Fig. 1. In the figure, we give groundtruth cross-section curves of human left leg, right arm and torso in (a), (b) and (c), respectively, which are taken from a human body model created with polygon modelling and highlighted in red. Using standard ellipses to approximate these cross-section curves, we obtain those highlighted in blue in the figure. Comparing the red and blue curves in Fig. 1, we can conclude that very large errors are introduced by standard ellipses.

To tackle the above problem, we propose composite generalized elliptic curves to approximate cross-section curves accurately. Composite generalized elliptic curves contain generalized ellipses, generalized elliptic curves, and composite generalized elliptic segments. Generalized ellipses and generalized elliptic curves will be investigated in this section. Composite generalized elliptic segments will be developed in the following section.

Generalized ellipses are applicable to closed curves. Since open curves are also widely applied in geometric modeling and computer-aided design, generalized elliptic curves are proposed to approximate open curves.

Through rotation operations, cross-section curves can be changed to lie in one of $x-y, y-z$, and $x-z$ planes. Taking closed cross-section curves in the $x-y$ plane as an example, their generalized ellipses can be mathematically formulated as

$$
x(v)=a_{0}+\sum_{j=1}^{J}\left(a_{2 j-1} \cos 2 j \pi v+a_{2 j} \sin 2 j \pi v\right)
$$




$$
y(v)=b_{0}+\sum_{j=1}^{J}\left(b_{2 j-1} \sin 2 j \pi v+b_{2 j} \cos 2 j \pi v\right)
$$

$$
z(v)-z_{c}=0
$$

where $0 \leq v \leq 1, a_{j}$ and $b_{j}(j=0,1,2,3, \cdots, 2 J)$ are unknown constants, which can be determined by fitting the generalized ellipse to the discrete points defining a closed cross-section curve.
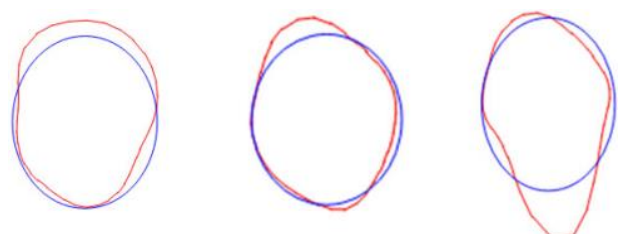

(a) Cross section curves of left leg

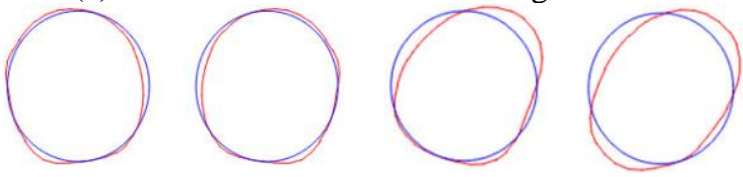

(b) Cross section curves of right arm
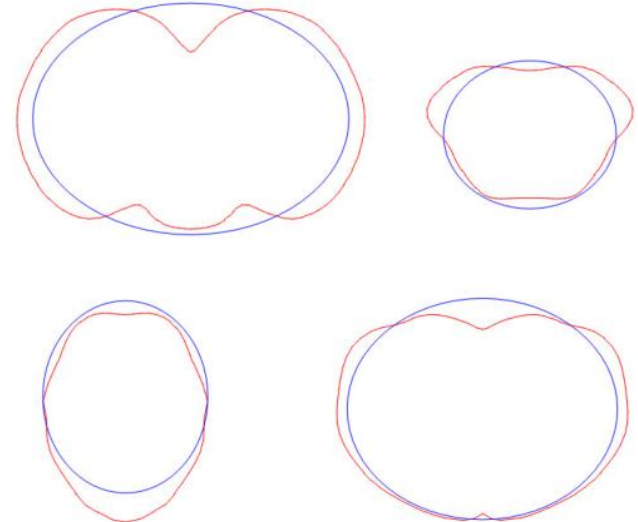

(c) Various cross section curves of torso

Fig. 1. Comparison between groundtruth cross-section curves of human body and approximated ellipses.

For a given closed curve defined by $n+1$ discrete points $\boldsymbol{X}_{i}=\left[\begin{array}{lll}x_{i} & y_{i} & z_{i}\end{array}\right]^{T}(i=$ $0,1,2, \cdots, n$ ), we first determine the total length $L$ of the closed curve and the length $L_{i}$ from the starting point $\boldsymbol{X}_{0}$ to the point $\boldsymbol{X}_{i}$. Then, we use $v_{i}=L_{i} / L$ to obtain the values of the parametric variable $v$ at these points. Finally, we obtain all the unknown constants by using Eq. (2) to fit these $n+1$ points.

For the groundtruth cross-section curves highlighted in red in Fig. 2, which are taken from a human bode model, we use standard ellipses and generalized ellipses to approximate them. The obtained results are shown in the same figure where the blue colour 
shows the curves obtained from standard ellipses, the green colour shows the curves obtained from generalized ellipses, and $J$ indicates the terms used in Eq. (2).

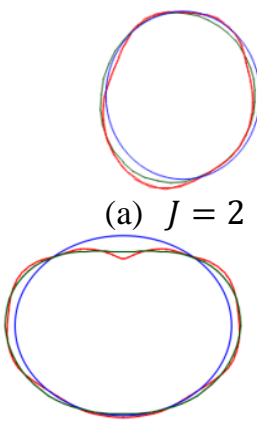

(d) $J=3$

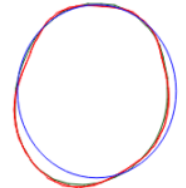

(b) $J=3$

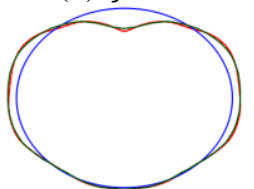

(e) $J=7$

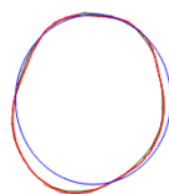

(c) $J=4$

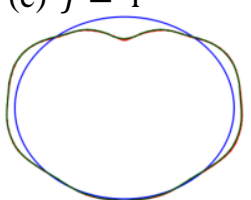

(f) $J=10$

Fig. 2. Comparison of curves created by standard and generalized ellipses.

Comparing the curves shown in Fig. 2, we can conclude: (1) there are very big differences between the standard ellipses and the groundtruth curves, (2) generalized ellipses approximate the groundtruth curves very well, (3) the groundtruth curves can be approximated accurately as the terms in Eq. (3) increase.

For closed curves approximated by Eq. (2), the starting point is same as the ending point. For open curves, the starting point is different from the ending point and Eq. (2) is not applicable. Therefore, we define the following generalized elliptic curves to approximate open curves in the $x-y$ plane.

$$
\begin{gathered}
x(v)=a_{0}+\sum_{j=1}^{J}\left(a_{2 j-1} \cos j \pi v+a_{2 j} \sin j \pi v\right) \\
y(v)=b_{0}+\sum_{j=1}^{J}\left(b_{2 j-1} \sin j \pi v+b_{2 j} \cos j \pi v\right) \\
z(v)-z_{c}=0
\end{gathered}
$$

where $0 \leq v \leq 1, a_{j}$ and $b_{j}(j=0,1,2,3, \cdots, 2 J)$ are unknown constants.

The unknown constants in Eq. (3) can be determined as follows. For discrete points $\boldsymbol{X}_{i}=\left[\begin{array}{lll}x_{i} & y_{i} & z_{i}\end{array}\right]^{T}(i=0,1,2, \cdots, n)$ of a curve, we calculate the total length $L$ of the curve and the length $L_{i}$ from the starting point $\boldsymbol{X}_{0}$ to the point $\boldsymbol{X}_{i}$, and obtain $v_{i}=L_{i} / L$. Then, we use $x_{0}=x\left(v_{0}\right)$ and $x_{n}=x\left(v_{n}\right)$ to determine two of the unknown constants $a_{j}(j=0,1,2, \cdots, 2 J)$ and $y_{0}=y\left(v_{0}\right)$ and $y_{n}=y\left(v_{n}\right)$ to determine two of the unknown constants $b_{j}(j=0,1,2, \cdots, 2 J)$ in Eq. (3). All the remaining unknown constants in Eq. (3) are determined by using it to fit the remaining $n-1$ points $\boldsymbol{X}_{i}$ ( $i=$ $1,2, \cdots, n-1)$. If tangential continuity is required, two more conditions of the first derivatives at the starting and ending points are used to determine two more unknown constants for each of the $x$ and $y$ components. Then, the remaining unknown constants are determined by curve fitting.

In Fig. 3, we use the generalized elliptic curve highlighted in red to approximate the original open curve highlighted in blue. The figure shows that when the total terms are 
not many, large errors are introduced as shown in Fig. 3(a). When more terms are used, the errors are reduced as shown by Fig. 3(d).

Table 1. Errors between the original open curve and open GEC.

\begin{tabular}{|c|c|c|c|c|c|c|}
\hline$J$ & 3 & 5 & 7 & 11 & 21 & 31 \\
\hline ErM & 2.2492 & 1.8117 & 1.2384 & 0.6711 & 0.3047 & 0.2197 \\
\hline ErA & 0.7459 & 0.4587 & 0.2519 & 0.1163 & 0.0460 & 0.0233 \\
\hline$J$ & 41 & 51 & 61 & 71 & 81 & 91 \\
\hline ErM & 0.1417 & 0.0900 & 0.0608 & 0.0369 & 0.0101 & $1.34 \times 10^{-4}$ \\
\hline ErA & 0.0138 & 0.0091 & 0.0072 & 0.0058 & $7.45 \times 10^{-4}$ & $4.35 \times 10^{-6}$ \\
\hline
\end{tabular}

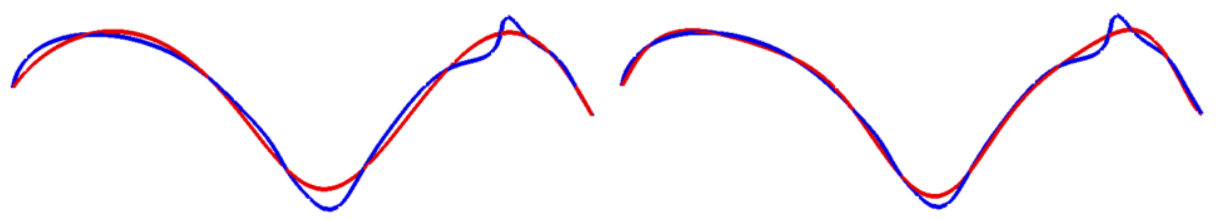

(a) $J=3$

(b) $J=5$

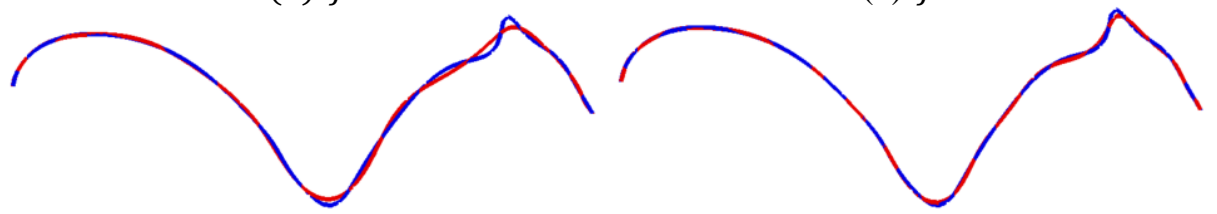

(c) $J=7$

(d) $J=11$

Fig. 3. Comparison between the original curve and generalized elliptic curve.

In Table 1, we give a quantity comparison of the errors between the original open curve and the generalized elliptic curve. In the table, ErM and ErA indicate the maximum error and average error, respectively. For the original open curve shown in Fig. 3, the maximum error reduces more slowly than the average error. Only when many terms, i. e., $J=91$, are use used, small errors are obtained. It indicates the necessity of using composite generalized elliptic segments to reduce design variables and improve the accuracy of curve fitting, which will be investigated in the following section.

\section{Composite generalized elliptic segments (CGESs)}

For an open or closed curve with a complicated shape, using a single generalized elliptic curve or a single generalized ellipse to approximate it may require many terms in Eq. (2) or (3). In order to reduce design variables, raise computational efficiency, and improve interactive modelling performance, it is worthy of developing a more efficient algorithm to cope with open and closed curves with complicated shapes and the corresponding surface reconstruction.

A complicated open and closed curve can be decomposed into several simpler ones. In order to approximate these decomposed curve segments, we use Eq. (3) to approximate each of them and name each segment as a composite generalized elliptic segment. 
As an example, we consider how to use two composite generalized elliptic segments to approximate an open curve with a complicated shape highlighted in blue shown in Fig. 4(a). For the curves consisting of more than two composite generalized elliptic segments, the treatment is the same.

Firstly, we segment the blue open curve in Fig. 4(a) into two separate curves ABC and CDE shown in Figs. 4(b) and 4(c), respectively. With Eq. (3) and the method of determining its unknown constants given in Section 3, we can determine the two composite generalized elliptic segments highlighted in red in Figs. 4(d), 4(e), and 4(f). Then, we use one single generalized elliptic curve to approximate the open curve and depict the obtained curve highlighted in pink in Figs. 4(g), 4(h) and 4(i). In the figure, $J$ indicates the terms defined in Eq. (3).

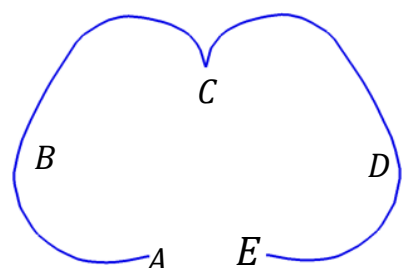

(a)

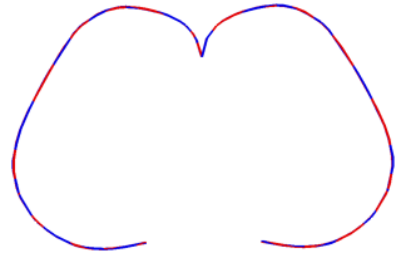

(d) $J=2$

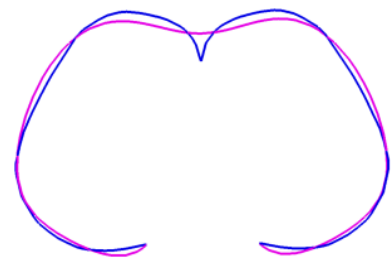

(g) $J=5$

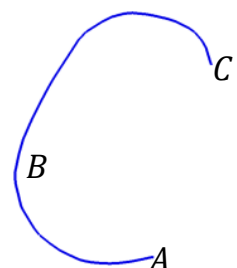

(b)

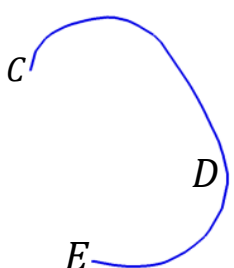

(c)
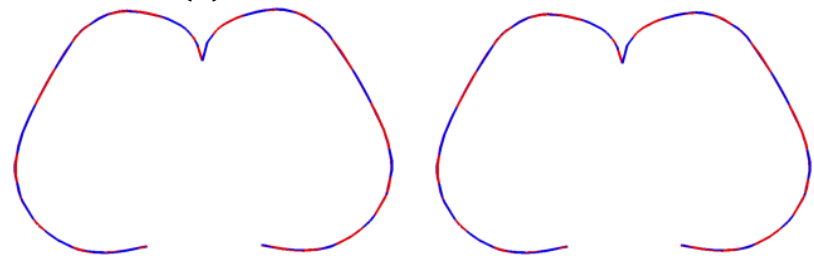

(e) $J=4$

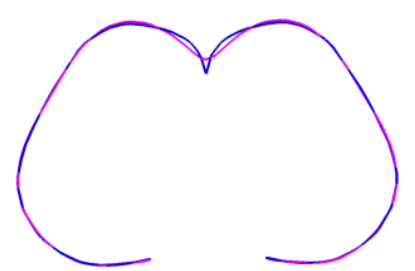

(h) $J=9$

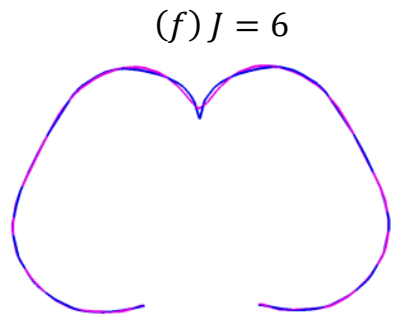

(i) $J=13$

Fig. 4. Comparison of curve approximation between two CGESs and one GEC.

First, we investigate the accuracy of using two composite generalized elliptic segments and one single generalized elliptic curve when using almost the same number of unknown constants. The unknown constants used by two composite generalized elliptic segments and one single generalized elliptic curve shown in second and third rows of Fig. 4 are almost the same. For example, the total unknown constants for two composite generalized elliptic segments shown in Fig. 3(d) are $(2 \times 2+1) \times 4=20$, and the total unknown constants for the generalized elliptic curve shown in Fig. $4(\mathrm{~g})$ are $(5 \times 2+$ 
1) $\times 2=22$. In spite of almost the same number of unknown constants, the two composite generalized elliptic segments shown in Fig. 4(d) approximate the open curve accurately. In contrast, very large errors are caused by the generalized elliptic curve shown in Fig. 4(g), indicating that composite generalized elliptic segments are much more accurate than generalized elliptic curves.

Next, we set the errors of the two composite generalized elliptic segments to be roughly same as the errors of the generalized elliptic curve, and compare the number of the unknown constants in Table 2 where ErM and ErA indicate the maximum and average errors between the groundtruth curve and the approximated ones. The total unknown constants for $J=5$ of the two composite generalized elliptic segments are $(5 \times 2+1) \times 4=44$. In contrast, the total unknown constants for $J=25$ of the generalized elliptic curve are $(25 \times 2+1) \times 2=102$. In order to achieve roughly same maximum and average errors, the total unknow constants of the generalized elliptic curve are 2.32 times of the two composite generalized elliptic segments. It indicates that using multiple composite generalized elliptic segments to approximate a complicate curve can noticeably reduce the design variables in comparison with using a single generalized elliptic curve.

Table 2. Comparison of errors between two CGESs and one GEC.

\begin{tabular}{|c|c|c|c|c|c|c|c|c|}
\hline & \multicolumn{3}{|c|}{ Composite generalized elliptic segments } & \multicolumn{4}{|c|}{ Generalized elliptic curve } \\
\hline & $J=5$ & $J=7$ & $J=15$ & $J=39$ & $J=25$ & $J=40$ & $J=59$ & $J=79$ \\
\hline ErM & 0.13 & 0.09 & 0.04 & $1.00 \times 10^{-5}$ & 0.49 & 0.23 & 0.08 & $5.30 \times 10^{-5}$ \\
\hline ErA & 0.04 & 0.03 & 0.01 & $3.08 \times 10^{-6}$ & 0.06 & 0.03 & 0.01 & $3.57 \times 10^{-6}$ \\
\hline
\end{tabular}

\section{Composite generalized elliptic curve-based surface reconstruction}

Since open and closed curves are described with generalized ellipses, generalized elliptic curves, and composite generalized elliptic segments, surface reconstruction is divided into generalized ellipse-based, generalized elliptic curve-based, and composite generalized elliptic segment-based, accordingly.

\subsection{Generalized ellipse or generalized elliptic curve-based surface reconstruction}

If a surface is reconstructed from $M$ generalized ellipses or generalized elliptic curves $\boldsymbol{X}_{m}(v)=\left[\begin{array}{lll}x_{m}(v) & y_{m}(v) & z_{m}(v)\end{array}\right]^{T}(m=0,1,2, \cdots, M)$, the surface can be mathematically formulated as

$$
\boldsymbol{S}(u, v)=\sum_{m=0}^{M} u^{m} \boldsymbol{G}_{m}(v)
$$


where $\boldsymbol{G}_{m}(v)(m=0,1,2, \cdots, M)$ are unknown vector-valued functions, which can be determined by interpolating the $M+1$ generalized ellipses or generalized elliptic curves.

When two surfaces are to be connected together with up to the tangential continuity, we can obtain the position function and first partial derivative function on the shared boundary curve from the surface, which has already been reconstructed. Then we can use the position function and first partial derivative function together with generalized ellipses or generalized elliptic curves to be interpolated to reconstruct a new surface.

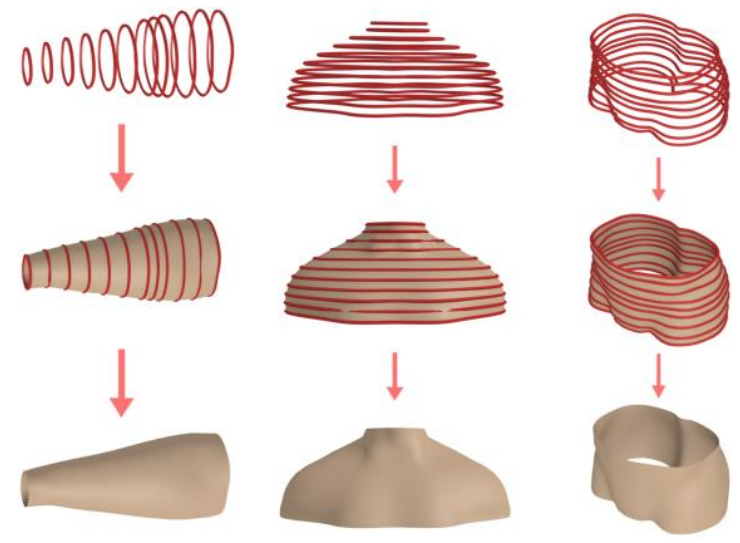

(a)
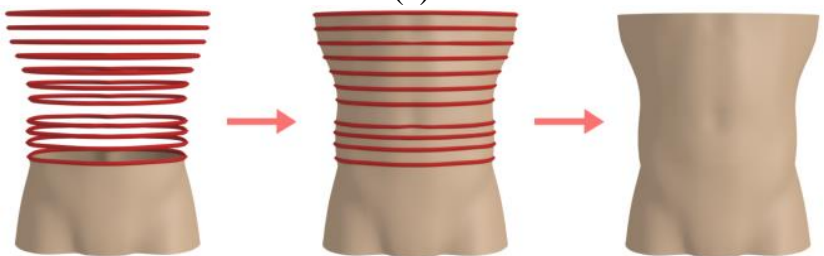

(b)

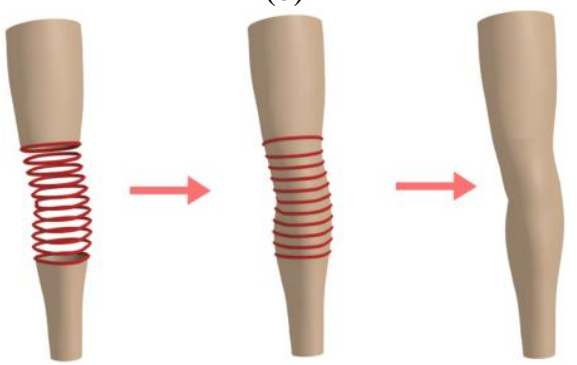

(c)

Fig. 5. Surfaces reconstructed from generalized ellipses.

With the above method, we reconstruct some surfaces from generalized ellipses and depict the reconstructed surfaces in Fig. 5. In the figure, (a) shows the surfaces totally determined by generalized ellipses, (b) shows the surface interpolating generalized ellipses and smoothly connecting to an adjacent surface, and (c) shows the surface interpolating generalized ellipses and smoothly connecting to two adjacent surfaces. 


\subsection{Composite generalized elliptic segment-based surface reconstruction}

Whether the curves are converted into generalized ellipses, generalized elliptic curves or composite generalized elliptic segments, they must be divided into the same number of curve segments if a surface is to be constructed from them. Therefore, when a generalized ellipse or a generalized elliptic curve is to be combined with other composite generalized elliptic segments to reconstruct a surface, the generalized ellipse or generalized elliptic curve should be firstly divided into the same segments as those of composite generalized elliptic segments. For example, if we are required to reconstruct a surface from three composite generalized elliptic segments and a single closed generalized ellipse, we divide the generalized ellipse into three curve segments .

The continuity between different surface patches along the $v$ parametric direction can be achieved by taking the mathematical expressions for $u$ parametric variable of the adjacent two surface patches to be the same form. In the following, we indicate that the surface patches reconstructed in this way can maintain the required continuity.

For $I+1$ curves each of which consists of $J+1$ segments, we use vector-valued position functions $\boldsymbol{X}_{i j}(i=0,1,2, \cdots, I ; j=0,1,2, \cdots, J)$ to represent the $j^{\text {th }}$ segment of the $i^{\text {th }}$ curve. Here $\boldsymbol{X}_{i j}$ has the three components $x_{i j}, y_{i j}$ and $z_{i j}$. Since $z_{i j}$ takes an identical value for all segments of a same curve, the continuity for this component is always ensured. Therefore, the following treatment is for $x$ and $y$ components. Here we only consider positional and tangential continuities. Of course, the treatment discussed here is also suitable for higher order continuities.

If up to tangential continuity is considered, both positional and tangential continuities at the joint between the $j^{\text {th }}$ segment $\boldsymbol{X}_{i j}$ and $(j+1)^{t h}$ segment $\boldsymbol{X}_{i j+1}$ should be achieved when constructing these curve segments. That is

$$
\begin{aligned}
& \boldsymbol{X}_{i j}\left(v=v_{i j}\right)=\boldsymbol{X}_{i j+1}\left(v=v_{i j}\right) \\
& \left\{\frac{\partial \boldsymbol{X}_{i j}}{\partial v}\right\}_{v=v_{i j}}=\left\{\frac{\partial \boldsymbol{X}_{i j+1}}{\partial v}\right\}_{v=v_{i j}} \\
& (i=0,1,2,3, \cdots, I ; j=1,2,3, \cdots, J-1)
\end{aligned}
$$

If the curves are closed, the positional and tangential continuities at the closure should also be introduced which lead to the additional equations below

$$
\begin{gathered}
\boldsymbol{X}_{i J}(v=2 \pi)=\boldsymbol{X}_{i 0}(v=0) \\
\left\{\frac{\partial \boldsymbol{X}_{i J}}{\partial v}\right\}_{v=2 \pi}=\left\{\frac{\partial \boldsymbol{X}_{i 0}}{\partial v}\right\}_{v=0} \\
(i=0,1,2,3 \ldots, I)
\end{gathered}
$$

If $J+1$ surface patches are constructed from the above $I+1$ curves, we take their surface functions to be

$$
\begin{gathered}
\boldsymbol{S}_{j}(u, v)=\sum_{m=0}^{I} u^{m} \overline{\boldsymbol{X}}_{m j}(v) \\
(j=0,1,2,3, \cdots, J)
\end{gathered}
$$

where $\overline{\boldsymbol{X}}_{m j}(v)(m=0,1,2,3, \ldots, I ; j=0,1,2,3, \ldots, J)$ are unknown functions.

At the position $u_{i}$ of the $i^{\text {th }}$ curve segment, the $j^{\text {th }}$ surface patch $\boldsymbol{S}_{j}(u, v)=$ $\sum_{m=0}^{I} u^{m} \overline{\boldsymbol{X}}_{m j}(v)$ and the $(j+1)^{t h}$ surface patch $\boldsymbol{S}_{j+1}(u, v)=\sum_{m=0}^{I} u^{m} \overline{\boldsymbol{X}}_{m j+1}(v)$ should pass through the $j^{\text {th }}$ and $(j+1)^{\text {th }}$ curve segments, respectively, i. e., 


$$
\begin{gathered}
\sum_{m=0}^{I} u_{i}^{m} \overline{\boldsymbol{X}}_{m j}(v)=\boldsymbol{X}_{i j}(v) \\
\sum_{m=0}^{I} u_{i}^{m} \overline{\boldsymbol{X}}_{m j+1}(v)=\boldsymbol{X}_{i j+1}(v) \\
(i=1,2,3, \ldots, I)
\end{gathered}
$$

Expanding Eqs. (6), (7) and (8) and rewriting them into the form of matrix, we obtain the following mathematical expressions from Eqs. (6) and (8) for open curves and from Eqs. (6), (7) and (8) for closed curves

$$
\begin{gathered}
{\left[R_{j}\left(u_{i m}\right)\right]\left\{\overline{\boldsymbol{X}}_{j}(v)\right\}=\left\{\boldsymbol{X}_{j}(v)\right\}} \\
{\left[R_{j+1}\left(u_{i m}\right)\right]\left\{\overline{\boldsymbol{X}}_{j+1}(v)\right\}=\left\{\boldsymbol{X}_{j+1}(v)\right\}}
\end{gathered}
$$

where $\left[R_{j}\left(u_{k m}\right)\right]$ and $\left[R_{j+1}\left(u_{k m}\right)\right]$ are $K \times K$ square matrices with the elements $u_{k m}=u_{k}^{m}, \quad\left\{\overline{\boldsymbol{X}}_{j}(v)\right\}=\left[\begin{array}{lllll}\overline{\boldsymbol{X}}_{0 j}(v) & \overline{\boldsymbol{X}}_{1 j}(v) & \overline{\boldsymbol{X}}_{2 j}(v) & \cdots & \overline{\boldsymbol{X}}_{I j}(v)\end{array}\right]^{T}, \quad\left\{\overline{\boldsymbol{X}}_{j+1}(v)\right\}=$ $\left[\begin{array}{llllll}\overline{\boldsymbol{X}}_{0 j+1}(v) & \overline{\boldsymbol{X}}_{1 j+1}(v) & \overline{\boldsymbol{X}}_{2 j+1}(v) & \cdots & \overline{\boldsymbol{X}}_{I j+1}(v)\end{array}\right]^{T} \quad, \quad\left\{\boldsymbol{X}_{j}(v)\right\}=$

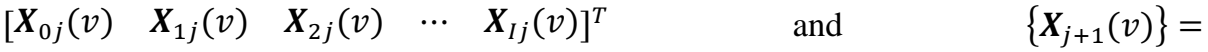
$\left[\begin{array}{lllll}\boldsymbol{X}_{0 j+1}(v) & \boldsymbol{X}_{1 j+1}(v) & \boldsymbol{X}_{2 j+1}(v) & \cdots & \boldsymbol{X}_{I j+1}(v)\end{array}\right]^{T}$ are the vectors with $K$ elements.

Using $\left[R_{j}\left(u_{i m}\right)\right]^{-1}$ and $\left[R_{j+1}\left(u_{i m}\right)\right]^{-1}$ to indicate the inverse matrices of $\left[R_{j}\left(u_{i m}\right)\right]$ and $\left[R_{j+1}\left(u_{i m}\right)\right]$, respectively, left multiplying both sides of the first equation of Eq. (9) by $\left[R_{j}\left(u_{i m}\right)\right]^{-1}$ and left multiplying both sides of the second equation of Eq. (9) by $\left[R_{j+1}\left(u_{i m}\right)\right]^{-1}$, we obtain the unknown functions with the following equations

$$
\begin{gathered}
\left\{\overline{\boldsymbol{X}}_{j}(v)\right\}=\left[\boldsymbol{R}_{j}\left(u_{i m}\right)\right]^{-1}\left\{\boldsymbol{X}_{j}(v)\right\} \\
\left\{\overline{\boldsymbol{X}}_{j+1}(v)\right\}=\left[\boldsymbol{R}_{j+1}\left(u_{i m}\right)\right]^{-1}\left\{\boldsymbol{X}_{j+1}(v)\right\} \\
(j=0,1,2,3, \ldots, J)
\end{gathered}
$$

From Eq. (8), we know that $\left[R_{j}\left(u_{i m}\right)\right]^{-1}$ and $\left[R_{j+1}\left(u_{i m}\right)\right]^{-1}$ are identical which can be written as

$$
\boldsymbol{R}=\left[R_{j}\left(u_{i m}\right)\right]^{-1}=\left[R_{j+1}\left(u_{i m}\right)\right]^{-1}=\left[R_{i j}\right]
$$

where $R_{i j}(i=0,1,2,3, \cdots, I ; j=0,1,2,3, \cdots, I)$ are the elements of the square matrix $\boldsymbol{R}$.

With Eq. (11), we can obtain the mathematical expressions of the elements in vectors $\overline{\boldsymbol{X}}_{j}(v)$ and $\overline{\boldsymbol{X}}_{j+1}(v)$

$$
\begin{aligned}
& \overline{\boldsymbol{X}}_{l j}(v)=\sum_{i=0}^{I} R_{l i} \boldsymbol{X}_{i j}(v) \\
& \overline{\boldsymbol{X}}_{l j+1}(v)=\sum_{i=0}^{I} R_{l i} \boldsymbol{X}_{i j+1}(v) \\
& (l=0,1,2,3, \ldots, I)
\end{aligned}
$$

Substituting the first of Eq. (12) into $\boldsymbol{S}_{j}(u, v)=\sum_{m=0}^{I} u^{m} \overline{\boldsymbol{X}}_{m j}(v)$ and the second of Eq. (12) into $\boldsymbol{S}_{j+1}(u, v)=\sum_{m=0}^{I} u^{m} \overline{\boldsymbol{X}}_{m j+1}(v)$, the position functions of the $j^{t h}$ and $(j+1)^{\text {th }}$ surface patches are found to be 


$$
\begin{gathered}
\boldsymbol{S}_{j}(u, v)=\sum_{m=0}^{I} u^{m} \sum_{i=0}^{I} R_{m i} \boldsymbol{X}_{i j}(v) \\
\boldsymbol{S}_{j+1}(u, v)=\sum_{m=0}^{I} u^{m} \sum_{i=0}^{I} R_{m i} \boldsymbol{X}_{i j+1}(v)
\end{gathered}
$$

From Eq. (13), we can calculate the first partial derivatives of the $j^{\text {th }}$ and $(j+1)^{t h}$ surface patches with respect to the parametric variable $v$. Considering the positional and tangential continuity conditions (5) and (6) of $\boldsymbol{X}_{i j}(v)$ and $\boldsymbol{X}_{i j+1}(v)$ at their joints $v_{i j}$, we find

$$
\begin{gathered}
\boldsymbol{S}_{j}\left(u, v_{i j}\right)=\boldsymbol{S}_{j+1}\left(u, v_{i j}\right) \\
\left\{\frac{\partial \boldsymbol{S}_{j}(u, v)}{\partial v}\right\}_{v=v_{i j}}=\left\{\frac{\partial \boldsymbol{S}_{j+1}(u, v)}{\partial v}\right\}_{v=v_{i j}}
\end{gathered}
$$

Equation (14) indicates that along the shared boundary curve between the $j^{\text {th }}$ and $(j+1)^{t h}$ surface patches, both positional and tangential continuities are guaranteed.

In Fig. 6, we use the above method to reconstruct a surface from three composite generalized elliptic segments. It demonstrates the effectiveness of the above method.
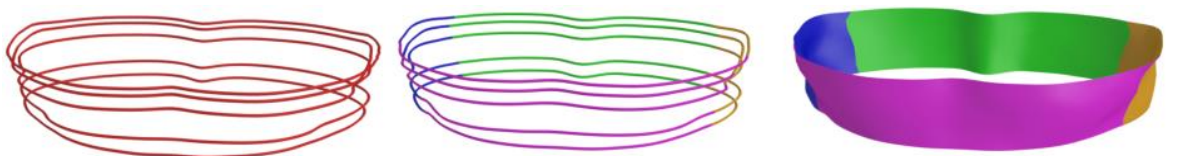

Fig. 6. A surface reconstructed from three composite generalized elliptic segments.

\subsection{Human body model reconstruction with composite generalized elliptic curves}

With the method developed above, the reconstruction of a human body model starts from obtaining cross-section curves shown in Fig. 7(a). These cross-section curves are converted into composite generalized elliptic curves. Then, the corresponding part models of the human body are reconstructed from the composite generalized elliptic curves and shown in Fig. 7(b).

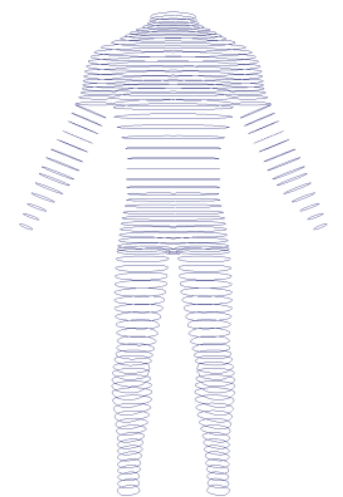

(a)

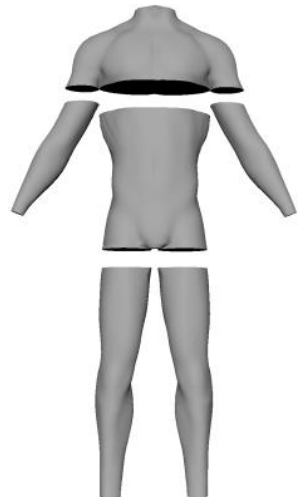

(b)

Fig. 7. Cross-section curves and part models of a human body. 
For this example of human body model reconstruction, all the part models share the same boundary curves. When these part models are reconstructed, they are naturally connected together as shown in Fig. 8 where Fig. 8(a) shows the reconstructed 3D model with cross section curves, and Fig. 8(b) shows the rendered 3D model.

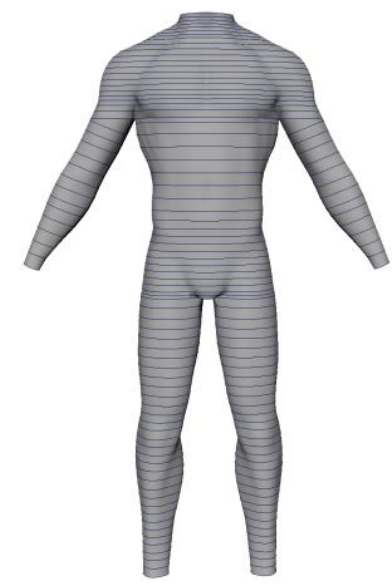

(a)

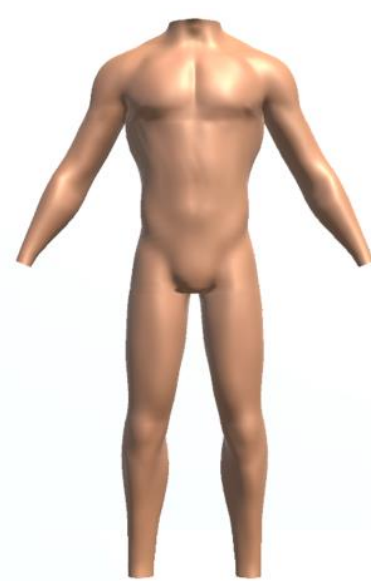

(b)

Fig. 8. Surface reconstruction of a human body model from composite generalized elliptic curves.

\section{Conclusions}

In this paper, we have proposed generalized ellipses, generalized elliptic curves, composite generalized elliptic segments to approximate open and closed curves, demonstrate their effectiveness and accuracy, and present mathematical expressions of surface reconstruction from composite generalized elliptic curves.

Compared to the sweep-based human deformation approach proposed in [12], the method proposed in this paper approximates cross-section curves accurately and avoids the use of a displacement map, leading to accurate and detailed reconstruction of 3D models.

Unlike polygon models, which involve a lot of design variables (vertices), the proposed method representing 3D models with analytical mathematical expressions involves much fewer design variables. It brings in the advantages of low storage costs, fast network transmissions, and easy generation of 3D models with arbitrary resolutions, which is suitable for many applications such as level of detail in online games.

\section{Acknowledgements}

This research is supported by the PDE-GIR project which has received funding from the European Union Horizon 2020 research and innovation programme under the Marie Skodowska-Curie grant agreement No 778035. 


\section{References}

1. Fuchs, H., Kedem, Z.M., Uselton, S.P.: Optimal surface reconstruction from planar contours. Communications of the ACM 20(10), 693-702 (1977).

2. Cook, P.N., Batnitzky, S., Lee, K.R., Levine, E., Price, H.I.: Three-dimensional reconstruction from serial sections for medical applications. Proceedings of SPIE 283, 98-105 (1981).

3. Boissonnat, J.D.: Shape reconstruction from planar cross sections. Computer Vision, Graphics, and Image Processing 44(1), 1-29 (1988).

4. Dierckx, P. Suetens, P., Vandermeulen, D.: An algorithm for surface reconstruction from planar contours using smoothing splines. Journal of Computational and Applied Mathematics 23, 367-388 (1988).

5. Meyers, D., Skinner, S., Sloan, K.: Surfaces from Contours. ACM Transactions on Graphics 11(3), 228-258, (1992).

6. Xu, M., Tang, Z.: Surface reconstruction for cross sectional data. Journal of Computer Science \& Technology 11(5), 471-479 (1996).

7. Bajaj, C.L., Coyle, E.J., Lin, K.N.: Arbitrary topology shape reconstruction from planar cross sections. Graphical Models and Image Processing 1996; 58(6):524-43.

8. Cong, G., Parvin, B.: An Algebraic Solution to Surface Recovery from Cross-Sectional Contours. Graphical Models and Image Processing 61, 222-243 (1999).

9. Cong, G., Parvin, B.: Robust and Efficient Surface Reconstruction from Contours. The Visual Computer 17, 199-208 (2001).

10. Knopf, G.K., Al-Naji, R.: Adaptive reconstruction of bone geometry from serial cross-sections. Artificial Intelligence in Engineering 15, 227-239 (2001).

11. Hyun, D.-E., Yoon, S.-H., Kim, M.-S., Jüttler, B.: Modeling and deformation of arms and legs based on ellipsoidal sweeping. In Proceedings of $11^{\text {th }}$ Pacific Conference on Computer Graphics and Applications, pp. 204-212. IEEE Computer Society (2003).

12. Hyun, D.-E., Yoon, S.-H., Chang, J.-W., Kim, M.-S., Jüttler, B.: Sweep-based human deformation. The Visual Computer 21, 542-550 (2005).

13. Akkouche, S. Galin, E.: Implicit surface reconstruction from contours. The Visual Computer 20, 392-401, 2004.

14. Park, H.: A hybrid approach to smooth surface reconstruction from 2-D cross sections. The International Journal of Advanced Manufacturing Technology 25, 1130-1136 (2005).

15. Liu, L., Bajaj, C., Deasy, J.O., Low, D.A., Ju, T.: Surface reconstruction from non-parallel curve networks. Computer Graphics Forum 27(2), 155-163 (2008).

16. Zou, M., Holloway, M., Carr, N., Ju, T.: Topology-constrained surface reconstruction from cross-sections. ACM Transactions on Graphics 34(4), Article No. 128: 1-10 (2015).

17. Holloway, M., Grimm, C., Ju, T.: Template-based surface reconstruction from cross-sections. Computers \& Graphics 58, 84-91 (2016).

18. Sharma, O., Agarwal, N.: Signed distance based 3D surface reconstruction from unorganized planar cross-sections. Computers \& Graphics 62, 67-76 (2017).

19. Zhong, D.-Y., Wang, L.-G., Jia, M.-T., Bi, L., Zhang, J.: Orebody Modeling from NonParallel Cross Sections with Geometry Constraints. Minerals 9(4), 229:1-17 (2019). 